\title{
EEG Mapping during Quiet and Sensory-Conflicted Stance
}

\section{Bozhidar Dimitrov $^{a}$, Katerina Stambolieva ${ }^{b}$ and Plamen Gatev ${ }^{b}$}

${ }^{a}$ Inst. of Population and Human Studies, BAS, Acad. G. Bonchev Str., BI. 6, Sofia 1113, Bulgaria

${ }^{b}$ Institute of Neurobiology, BAS, Acad. G. Bonchev Str., BI. 23, Sofia 1113, Bulgaria

ABSTRACT: The body balance is dependent upon quantity, quality and congruency of sensory inputs from three major sources. When compromised, i.e., introducing a sensory conflict, a complex sensory and cognitive integration and reweighing is to be executed in cerebral cortex in order to maintain balance. We have investigated the cortical map changes in ten different conditions of quiet standing by means of calculating the EEG power spectral density distribution and connectivity of cortical areas between them and prefrontal dorsolateral cortex. The prevailing role of the visual sensory input has been accentuated and the cortical regulation of posture and balance was demonstrated by contemporary techniques. The results suggested that the left dorsolateral prefrontal cortical area play a role in solving the sensory conflict.

KEYWORDS: Electroencephalography, brain maps, postural control, stance, sensory conflict

\section{Introduction}

The problem with upright stance in humans began the moment they changed from the more stable-and easy to maintain-quadruped position onto the feeble and risky two-legs-only. As George Orwell once has put it, although in quite another vein: "two legs - good, four legs - better." Following the immeasurably prolonged course of evolution, aided by much training and upgraded with cognitive constructs, these days the stable upright stance seems to be of no concern for the healthy bearer despite instability due to the highly positioned center of gravity. The comfortable condition is being achieved due to the constant and finely tuned interplay between many multilayered regulatory hubs, connected with the posturokinetic apparatus and the nervous system. The major participants are: receptors in joints, muscles and skin; vestibular signals and visual input. Since the environmental surroundings change incessantly in their own often weird and unpredicted order (type of support area, illu- 
mination etc.) - all introducing sensory conflict due to an incongruence of information - the successful maintenance of stable posture depends on the reweighting of information inputs and adequate sensory integration. Recently, studies regarding the participation of cerebral cortex as a supreme regulator and coordinator have begun to appear. The cerebral cortex contributes to postural control by sensorimotor processing of postural instability, ${ }^{1}$ or modulation of postural responses via cortical response loops. $^{2,3}$ In this study we aimed at revealing the possible engagement of certain cortical areas in tasks with different complexity as regarding the altered involvement of some sensory channels. It has been found long ago that task difficulty increased cortical activity ${ }^{4}$ and that difficult postural tasks required more cognitive processing. ${ }^{5}$

\section{Methods}

Seven healthy volunteers (3 females, 4 males, mean age 36 years), were either sitting in an armchair or quietly standing on a stabilographic platform. The foot position while standing was with heels separated by $3 \mathrm{~cm}$ and forward inclination of $30^{\circ}$ for the toes. The duration of a recording session was $120 \mathrm{~s}$, randomly interspersed among 8 possible conditions with 2-3 min rests in between. In some positions the feet support was altered by a $10 \mathrm{~cm}$ polyurethane foam support and in other the head was kept maximally extended backwards. Thus, the established series are shown in Table 1 .

The electroencephalogram (EEG) was registered with 19 scalp electrodes attached on the scalp in accordance to the
10-20 of the IFCN on a 24 channel MITSAR EEG machine, with sampling rate of $500 \mathrm{~Hz}$. Following off-line editing and deleting of artifact-contaminated epochs the EEG underwent Fast-Fourier transform, enabling the creation of brain maps and coherence evaluation. We report here only partial amount of the vast data accumulated, mostly concerning the visual analyst contribution and the comparison between standing and sitting.

Table 1. Experimental series.

\section{Eyes Open, Sitting \\ 2 Eyes Closed, Sitting}

\begin{tabular}{|c|c|c|}
\hline 3 & $\begin{array}{l}\text { Eyes Open, } \\
\text { Stable Support }\end{array}$ & quiet stance \\
\hline 4 & $\begin{array}{l}\text { Eyes Closed, } \\
\text { Stable Support }\end{array}$ & absence of vision \\
\hline
\end{tabular}

\begin{tabular}{lll}
5 & $\begin{array}{l}\text { Eyes Open, } \\
\text { Foam Support }\end{array}$ & $\begin{array}{l}\text { altered } \\
\text { proprioception }\end{array}$ \\
6 & $\begin{array}{l}\text { Eyes Closed, } \\
\text { Foam Support }\end{array}$ & $\begin{array}{l}\text { altered propriocep- } \\
\text { tion + absence of } \\
\text { vision }\end{array}$ \\
\hline
\end{tabular}
7 Eyes Open, Sta- altered vestibular ble Support, information Head Extended
8 Eyes Closed, absence of vision + Stable Support, altered vestibular Head Extended information

\begin{tabular}{|c|c|c|}
\hline 9 & $\begin{array}{l}\text { Eyes Open, } \\
\text { Foam Support, } \\
\text { Head Extended }\end{array}$ & $\begin{array}{l}\text { altered propriocep- } \\
\text { tion + altered ves- } \\
\text { tibular information }\end{array}$ \\
\hline 10 & $\begin{array}{l}\text { Eyes Closed, } \\
\text { Foam Support, } \\
\text { Head Extended }\end{array}$ & $\begin{array}{l}\text { altered propriocep- } \\
\text { tion + altered ves- } \\
\text { tibular information }+ \\
\text { absence of vision }\end{array}$ \\
\hline
\end{tabular}




\section{Results and Discussion}

The body equilibrium has been found to be hampered by changes in the sensory inflow: the strongest standing balance destabilization has been found in series 10 (Fig.1, bottom right). Under these conditions the sensory conflict has achieved its utmost levels: shut-off of visual input, altered proprioception and reduced tactile sensitivity from feet receptors, altered vestibular information stemming from lowered otoliths sensitivity and altered vestibular canals' position. It also might be accentuated that softening of support via foam block has greater effect towards increasing body sway than that of head extension. Finally, eyes closed condition increased body sway - a fact, well known and established by many previous studies. ${ }^{6,7}$

The spectral power density in Delta, Theta, Alpha1, Alpha2, Beta and Gamma range of the EEG has been calculated. There occurred an increase in Alpha range with eyes closed and quiet stance on stable support (series 4). This is in accordance with previous studies, analyzing EEG activity while sitting or lying supine with eyes open (EO) vs. eyes closed (EC) (Fig.2, left panel). However, during standing with eyes closed vs. sitting we observed a well expressed diminution of the spectral power in Alpha1 band in the occipital cortical area. Standing with eyes open vs. sitting led to an increased Delta activity distribution over frontal areas, a decrease of Theta power in occipital area and increases in Beta and Gamma power in the frontal area (Fig.2, right panel). Our results suggest that there are clear changes in the cortical power distribution while standing compared to sitting, which affected more bands during EO vs. EC condition. During standing with EO AND altered somatosensory AND vestibular information a significant decrease in low frequencies (Delta and Theta power spectra) was noted. This constellation was preserved in EC standing position for Delta and Theta, whereas Alpha showed an increase in spectral power, especially over temporal and parietal associative areas, during standing on a foam support. Standing with eyes open and head extended (series 7) was accompanied by Alpha increase over frontal, central and parietal leads; with EC (series 8) this share did not change over central scalp areas. Our results are in line with increased Theta activation found in parietal area when visual and proprioceptive demands were increased. ${ }^{8}$

The EEG activity derived over all scalp areas was correlated with EEG activity over dorsolateral prefrontal cortex (lead Fp1) in conditions 3 to 10 . Fig.3 presents marked decrease in correlation with lengthening the distance to $\mathrm{Fp} 1$, valid for both EO and EC conditions.

Quite evident was the increased correlation between almost all areas and Fp1 in EC vs. EO series. In the most strenuous condition (10) a tendency for increased correlation was observed for parietal, temporal and occipital areas. There were increased correlations vs. Fp1 during standing compared to sitting in temporal area (EO, EC) and occipital area (EC) while decreases were observed for the central area (EO, EC) (Fig.3). The prefrontal area is the preferred site for evaluation, decision making and conflict solving, related to social and emotional entities. ${ }^{9}$ The role of prefrontal cortex in human equilibrium control was suggested by Mihara 
3

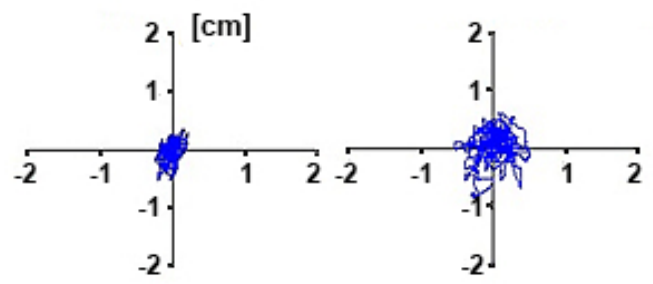

4

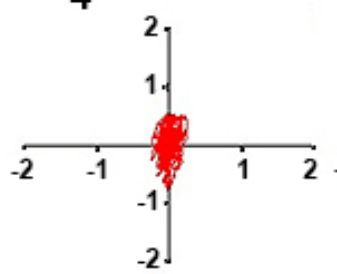

5

6

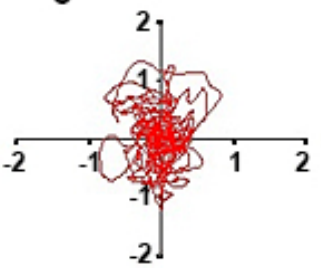

7

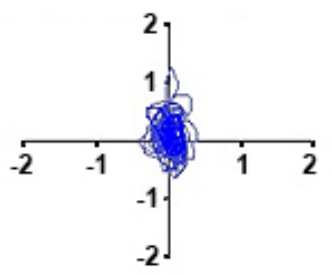

8

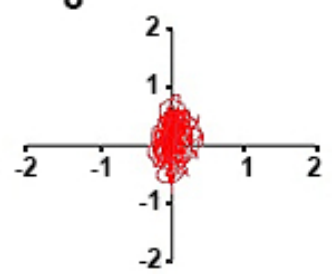

9

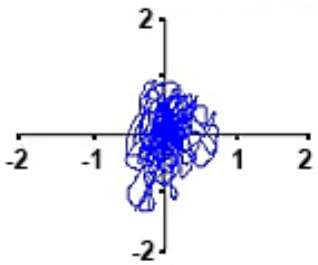

10

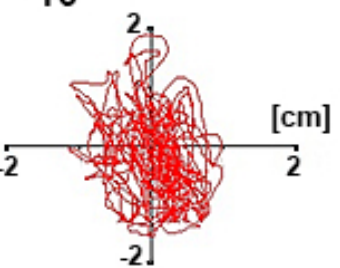

Figure 1: Body sway statokinesigram during upright standing. Upper row (blue) = Eyes Open; Bottom row (red) = Eyes Closed. (See Table 1 for numbered series order.)

EYES-CLOSED
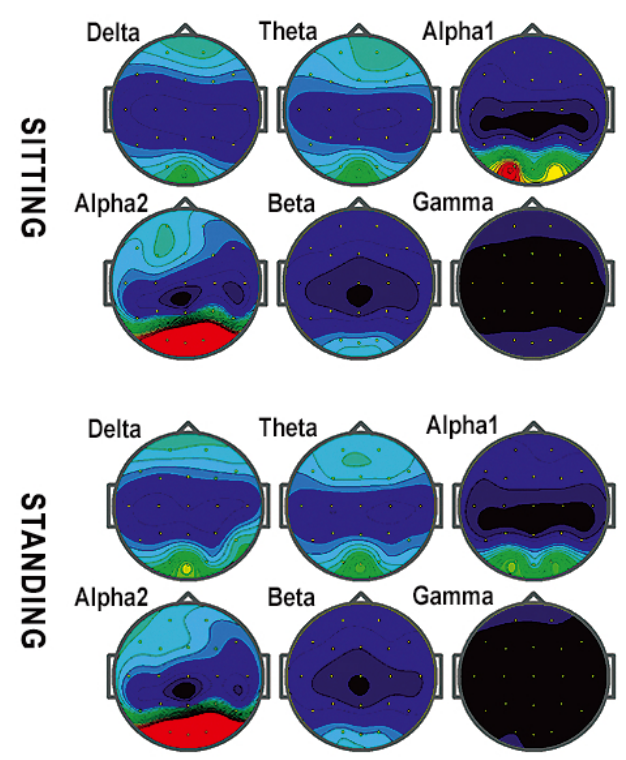

EYES-OPEN

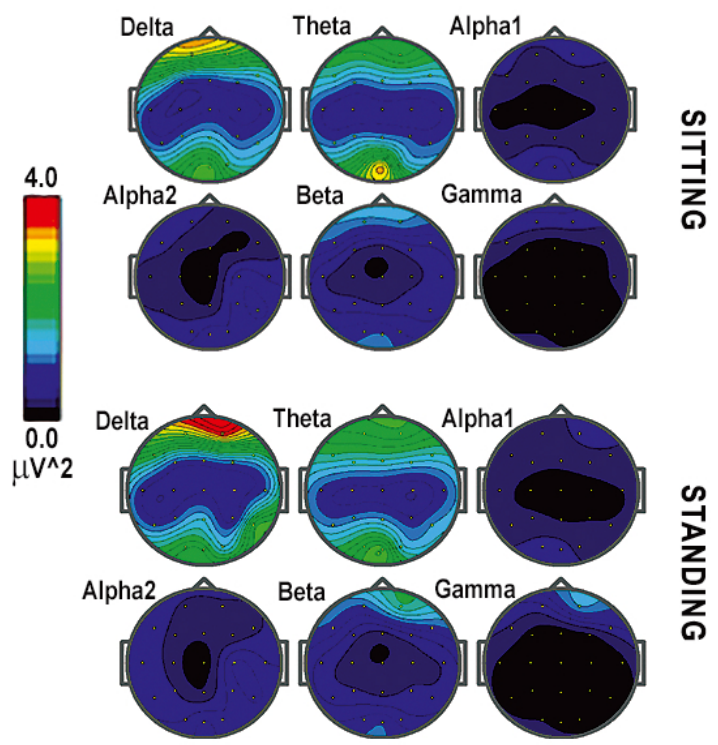

Figure 2: Brain maps of the absolute power spectral distribution for the frequency ranges: Delta (0.5-4Hz), Theta (4-8 Hz), Alpha1 (8-10 Hz), Alpha2 (10-12 Hz), Beta (12-30 Hz), Gamma (30-65 Hz). Eyes Closed (left panel): Sitting (upper rows) vs. Standing (lower rows); Eyes Open Sitting (upper rows) vs. Standing (lower rows). 


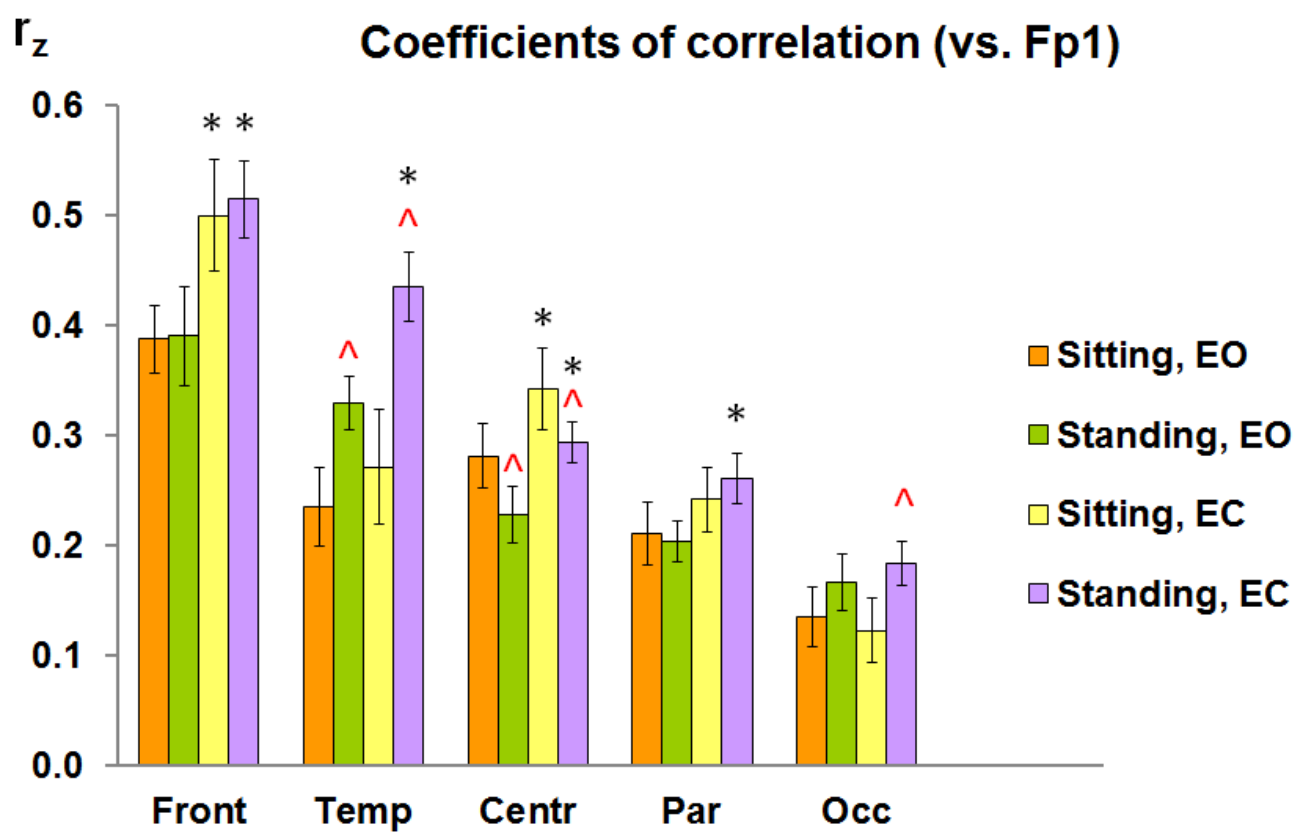

Figure 3: Data presented are means of Z-transformed correlation coefficients (rz) +/- SEM. *$p \leq 0.05$ - differences between EO/EC for each experimental series; ${ }^{\wedge}$ - $p \leq 0.05$ - differences for each experimental series vs. either series 3 (EO) or 4 (EC).
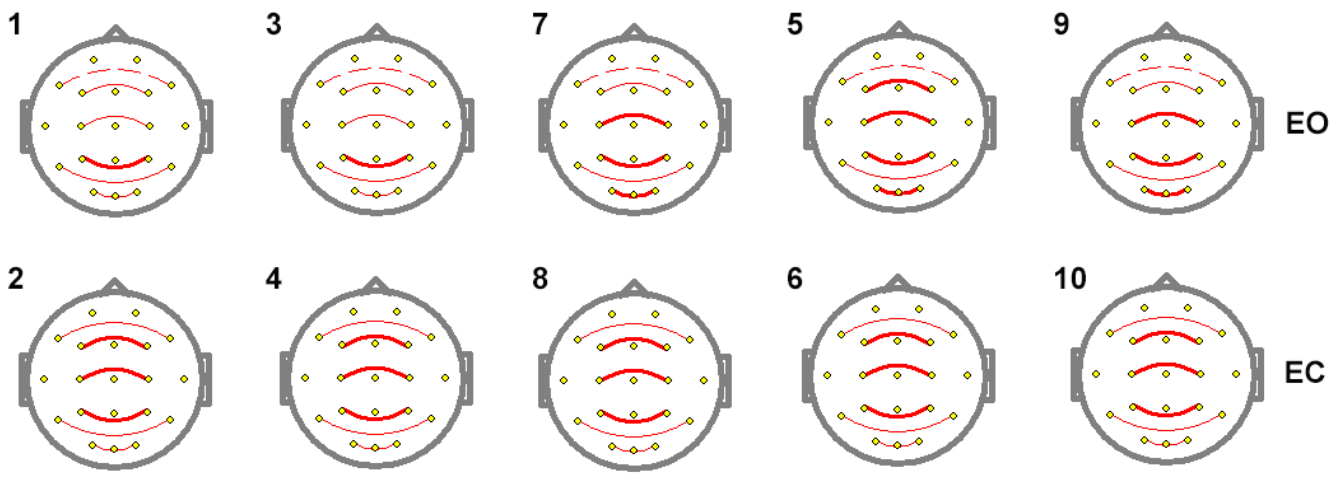

$-0.40$

0.60

0.80

$-0.40$

Figure 4: Left/right connectivity and differences between 10 separate conditions, as labeled within. The strength of coherence is proportional to the thickness of lines.

and Mayaki (2008)..$^{10}$ Our results implicate that this cortical area has a role in the solving of the sensory conflict during standing, as well. 
The features of left/right connectivity of EEG activity over cortical areas based on coherence are presented in Fig. 4. The results showed that EC compared to EO conditions offered more left/right coherence, especially in central and frontal areas, but there were no differences between the series. There were no differences between quiet standing compared to sitting both in EO and EC conditions.

The left/right connectivity, however, changes during sensory-conflicted stance with EO with an increase mostly expressed in central and occipital areas, which suggests that left/right symmetry of sensory information and standing balance play role in the sensory conflict solution, when visual input is available.

Quite recently, Thibault et al. $(2014)^{11}$ have studied with neuroimaging techniques the EEG changes in positions supine vs. inclined vs. sitting vs. posture and have found increased widespread high-frequency oscillatory activity in upright stance. Stressing the importance of posture as a determinant in the brain functioning they suggest that cognitive neuroscientists should always consider the influence of posture on brain dynamics. The results of Tse et al.(2013) ${ }^{12}$ also suggest a relationship between the amount of cortical activation and the task difficulty, based on calculations of changes in higher frequency bands (Beta/Sigma).

The results presented here suggest that cortical regulation of free stance in conditions of sensory conflict is dependent mostly on the participation of the visual input. The visual integration, being phylogenetically the youngest chain in the system of sensory analyzers, takes precedence and plays a leading role in the engagement of brain resources. Next, the changes in the EEG spectral power for different bands signal possible differences in cortical regulation of solving the problem of sensory integration caused by vestibular- and/or proprioceptive- dependent conflicting information. The changing amount of correlation between EEG activity from various cortical areas and the EEG activity vs. the left dorsolateral prefrontal cortex hints at the important role of this structure for solving of sensory conflict during maintaining of postural steadiness of balance.

Next, the changes in the EEG spectral frequency power for different bands signal possible differences in cortical regulation of solving the problem of sensory integration caused by vestibular- and/or proprioceptive- dependent conflicting information. The amount of correlation between EEG activity from various cortical areas and the EEG activity over the left dorsolateral prefrontal cortex hints at an important role of this structure for solving different forms of sensory conflict during stance.

\section{Acknowledgements}

The authors are thankful for the financial support provided by Grant TK 02/60 with the National Science Fund, Ministry of Education, Youth and Science, Republic of Bulgaria.

\section{References}

1. Slobounov S, Hallett M, Stanhope S, et al. Role of cerebral cortex in human postural control: an EEG study. Clin Neurophysiol. 2005 Feb;116(2):315-23. doi: 10.1016/j.clinph.2004.09.007. 
2. Jacobs JV, Horak FB. Cortical control of postural responses. J Neural Transm. 2007; 114(10):1339-48. doi: 10.1007/ s00702-007-0657-0.

3. Maki BE, Mcllroy WE. Cognitive demands and cortical control of human balance-recovery reactions. J Neural Transm. 2007; 114(10):1279-96. doi: 10.1007/s00702-007-0764-y.

4. Ouchi Y, Okada H, Yoshikawa E et al. Brain activation during maintenance of standing postures in human. Brain. 1999 Feb;122 (Pt 2):329-38. doi: 10.1093/ brain/122.2.329.

5. Teasdale N, Stelmach GE, Breunig A. Postural sway characteristics of the elderly under normal and altered visual and support surface conditions. J Gerontol. 1991 Nov; 46(6):B238-44. doi: 10.1093/geronj/46.6.B238.

6. Tanaka H, Nakashizuka M, Uetaki T, et al. The effects of visual input on postural control mechanisms: An analysis of center-of-pressure trajectories using the auto-regressive model. J Hum Ergol (Tokyo). 2000 Dec;29(1-2):15-25.

7. Stambolieva K, Diafas V, Bachev V, et al. Postural stability of canoeing and kayaking young male athletes during quiet stance. Eur J Appl Physiol. 2012 May;112(5):1807-15. doi: 10.1007/s004 21-011-2151-5.

8. Mizelle JC, Forrester L, Hallett $M$, et al. Electroencephalographic reactivity to unimodal and bimodal visual and proprioceptive demands in sensorimotor integration. Exp Brain Res. 2010 Jun;203(4): 659-70. doi:10.1007/s00221010-2273-8.

9. Miller EK, Cohen JD. An integrative theory of prefrontal cortex function. Annu Rev Neurosci. 2001;24:167-202. doi: 10.1146/annurev.neuro.24.1.167.

10. Mihara M, Miyai I, Hatakenaka M, et al. Role of the prefrontal cortex in human balance control. Neuroimage. 2008 Nov 1;43(2):329-36. doi: 10.1016/ j.neuroimage.2008.07.029.

11. Thibault, RT, Lifshitz $M$, Jones JM, et al. Posture alters human resting state. Cortex. 2014 Sep;58:199-205. doi: 10.1016/j.cortex.2014.06.014.

12. Tse YYF, Petrofsky JS, Berk L, et al. Postural sway and rhythmic electroencephalography analysis of cortical activation during eight balance training tasks. Med Sci Monit. 2013 Mar 8; 19:175-86. doi: 10.12659/MSM.883824. 